\title{
Capillary-Channeled Polymer (C-CP) Films as Processing Platforms for Protein Analysis by Matrix-Assisted Laser/Desorption Ionization Mass Spectrometry (MALDI-MS)
}

\author{
Jennifer J. Pittman, ${ }^{1}$ Benjamin T. Manard, ${ }^{1}$ Paul J. Kowalski, ${ }^{2}$ R. Kenneth Marcus ${ }^{1}$ \\ ${ }^{1}$ Department of Chemistry, Biosystems Research Complex, Clemson University, Clemson, SC 29634, USA \\ ${ }^{2}$ Bruker Daltonics, Inc., Billerica, MA, USA
}

\begin{abstract}
Polypropylene (PP) capillary-channeled polymer (C-CP) films have parallel, $\mu \mathrm{m}$-sized channels that induce solution wicking via capillary action. Efficient mass transport from the solution phase to the channel surface leads to adsorption of hydrophobic protein solutes. The basic premise by which C-CP films can be used as media to manipulate analyte solutions (e.g., proteins in buffer), for the purpose of desalting or chromatographic separation prior to MALDI-MS analysis is presented here. Cytochrome $c$ and myoglobin prepared in a Tris- $\mathrm{HCl}$ buffer, and ribonuclease A, lysozyme, and transferrin prepared in phosphate buffered saline (PBS), are used as the test solutions to demonstrate the desalting concept. Protein analysis is performed after deposition on a C-CP film with and without a water washing step, followed by spray deposition of a typical sinapinic acid matrix. Extracted MALDI mass spectra exhibit much improved signal-to-noise characteristics after water washing. A mixture of cytochrome $c$ and myoglobin $(2 \mu \mathrm{L}$ of $2.5 \mu \mathrm{M}$ each in Tris- $\mathrm{HCl}$ buffer) was applied, washed with water and spatially separated via simple capillary action (wicking) using a reversed-phase solvent composition of $0.1 \%$ trifluoroacetic acid (TFA) in 50:50 acetonitrile (ACN): $\mathrm{H}_{2} \mathrm{O}$. Subsequent application of sinapinic acid followed by imaging of the film using MALDI-MS reveals that as the protein solution is wicked down the film, separation occurs.
\end{abstract}

Key words: Capillary-Channeled Polymer (C-CP), Films, Thin layer chromatography (TLC), Matrix assisted laser desorption ionization (MALDI), Protein, Mass spectrometric imaging

\section{Introduction}

$\mathrm{T}$ hin layer chromatography (TLC) provides benefits over column chromatography methods due to the simplicity and versatility of the technique $[1,2]$. One of the primary downsides of TLC is the ambiguous nature of spot identification. Analytical determinations often require excision and/or dissolution prior to assay, resulting in non-quantitative recoveries, increased risk of contamination and analysis time, and analysis (solely) of removed spots. Online coupling of TLC with mass spectrometry detection began in the 1990s, involv-

Correspondence to: R. K. Marcus; e-mail: marcusr@clemson.edu ing a number of ion/atom/photon bombardment methods [3-5]. Key in each of these methods is the need to ablate analyte species that exist within the sorbent matrix. Therefore, the probe beam must penetrate the medium or some form of extraction/transfer of the solutes (i.e., blotting) from the medium must be employed. The advent of matrix-assisted laser desorption ionization mass spectrometry (MALDI-MS) provided further impetus for the TLC profiling of biomacromolecules [6-9]. MALDI-MS analysis generally involves extraction of solutes to the secondary sorbent followed by application of the MALDI matrix to affect co-crystallization. Direct MALDI from TLC plates has also been described, addressing the transfer issues [9]. 
Buffers typically employed in the processing of biological samples can be detrimental in MALDI (and electrospray ionization (ESI)) mass spectrometry, resulting in solute ionization suppression and formation of adducts that complicate spectral interpretation. The removal of buffer species (a.k.a., desalting) is achieved by use of a sorbent/chromatographic solid phase extraction (SPE) medium, followed by elution of proteins into solvents compatible with the ionization process $[10,11]$. This occurs de facto in TLC due to different migration rates of macromolecules and salts $[12,13]$.

Capillary-channeled polymer (C-CP) fibers have been explored in this laboratory for their use in protein chromatography and SPE [14-19]. C-CP fibers have several positive attributes towards macromolecular separations, most importantly enhanced mass transfer efficiencies that allow very rapid separations with high recoveries $[15,17]$. C-CP fibers can be extruded from a number of common polymers, affecting a range of surface chemistries with low materials cost. Relevant here, the $\mathrm{C}-\mathrm{CP}$ fibers have also been implemented in a micropipette tip format as a sorbent for desalting proteins prior to ESI-MS [18]. C-CPs can also be extruded in $\sim 5 \mathrm{~mm}$ wide film format, with parallel channels as presented in the electron micrographs of Figure 1. The channels $(\sim 5-40 \mu \mathrm{m}$ in width) create capillary forces that result in the ability to spontaneously move solutions along the film. Radial diffusion in the channels promotes efficient solute-surface interactions; effectively yielding parallel TLC channels. Thus, different from Lebrilla and coworkers who used a single open CE channel [20], the films present parallel channels of smaller dimension affording solute desalting and separations via wicking action rather than electrophoretic forces. In addition, there is no buffer/electrolyte residue in the ablation zone as in the case of the $\mathrm{CE}$ channel.

Demonstrated here is the concept of using C-CP films as processing platforms for protein analysis by MALDI-MS. Different from the aforementioned applications of TLCMALDI-MS, the proteins reside on the surface of the polymer channels; therefore no extraction step is necessary. Samples can be desalted or separated on C-CP films via wicking action in seconds. The long-term goal is to generate a phase that is more practical than conventional TLC plates, can be tailored to specific analytes, allows for low sample and solvent consumption $(\mu \mathrm{L})$, and can be imaged directly.

\section{Experimental}

\section{Chemicals and Reagents}

A working solution containing $2.5 \mu \mathrm{M}$ each of cytochrome $c$ and myoglobin, (Sigma Aldrich, St. Louis, MO, USA) was prepared in $100 \mathrm{mM}$ Tris-HCl (Teknova, Hollister, CA, USA ) and MilliQ water $(18.2 \mathrm{M} \Omega / \mathrm{cm})$ derived from a NANOpure Diamond Barnstead/Thermolyne Water System (Dubuque, IA, USA). Individual protein solutions of $2.5 \mu \mathrm{M}$ ribonuclease $A$ and transferrin were prepared in $150 \mathrm{mM}$ phosphate buffered saline (PBS) at $\mathrm{pH}$ 7.3. Protein separations were achieved using a 50:50 acetonitrile:water $\left(\mathrm{ACN}: \mathrm{H}_{2} \mathrm{O}\right)$ eluent. MALDI matrix solutions consisting of $15 \mathrm{mg} / \mathrm{mL}$ of sinapinic acid (Fisher Scientific, Pittsburgh, PA, USA) were prepared in $0.1 \%$ trifluoroacetic acid (TFA) (Sigma Adrich), 50:50 ACN: $\mathrm{H}_{2} \mathrm{O}$ (VWR, West Chester, PA).

\section{Film Preparation and Sample Spotting}

Polypropylene (PP) C-CP films were melt-extruded at the Clemson University School of Materials Science and Engineering (Clemson, SC, USA). Films are also available from a commercial source, Specialty and Custom Fibers, LLC (Clemson, SC, USA). Film segments were conditioned sequentially with water, methanol, and acetonitrile to remove any production residues. The films were attached via double-sided tape to a conventional steel MALDI target prior to application of the solutions. For all on-film analysis, $2 \mu \mathrm{L}$ of protein solution was spotted and allowed to dry $(<1 \mathrm{~min}$.) before any additional treatments occurred. Protein solution residues were washed by applying $9 \mu \mathrm{L}$ of DI- $\mathrm{H}_{2} \mathrm{O}$ to the loaded protein. After $5 \mathrm{~s}$, the water was pipetted back off of the film. In the case of the protein separation and film imaging (Figure 3), a $2 \mu \mathrm{L}$ aliquot of the working protein solution was applied to one end of a $2 \mathrm{~cm}$ long film and washed as previously described, with the separation of the
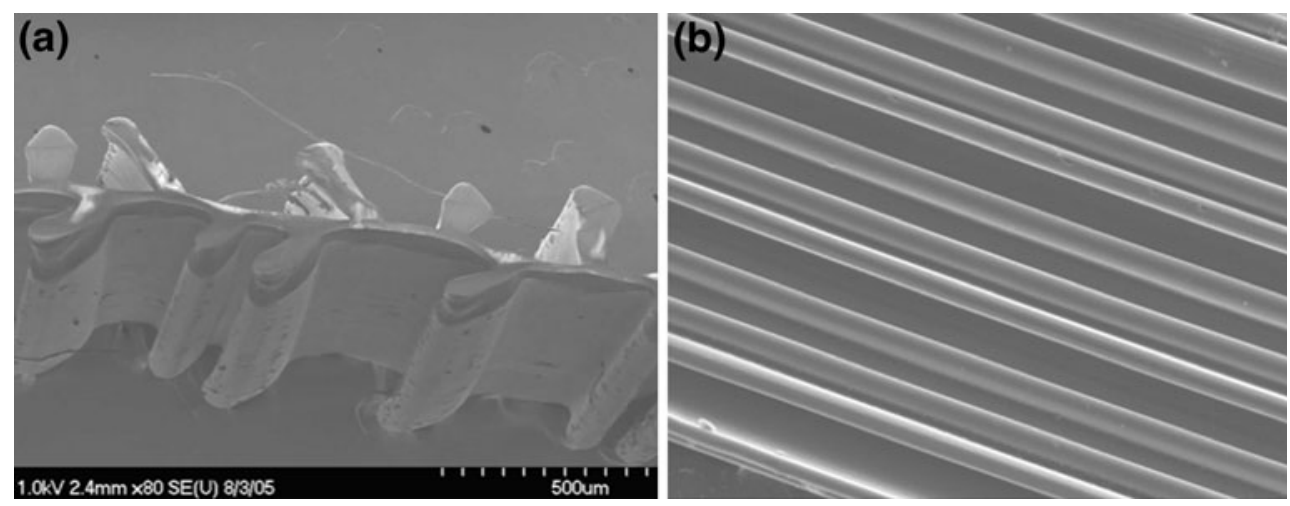

Figure 1. SEM micrographs of a polypropylene (PP) C-CP film; (a) cross section and (b) channel structure 
proteins initiated using $9 \mu \mathrm{L}$ of eluent, resulting in transport down the plurality of the channels. The respective solutions were dried under ambient conditions and the matrix solution applied using a chromatography reagent sprayer (Sigma Aldrich). Use of a sprayer (as opposed to wicking) ensures the integrity of the chromatographic separations.

\section{MALDI Analysis}

Analyte spectra were obtained on a Bruker Daltonics (Billerica, MA, USA) microflex LRF, MALDI-TOF mass spectrometer in the positive ion, linear mode. Instrument control and data processing occurred in the Compass (Bruker Daltonics) software environment. The microflex is equipped with a nitrogen laser $(337 \mathrm{~nm})$ operating at a pulse rate of $60 \mathrm{~Hz}$. Mass spectral acquisitions occurred using 200 laser shots at 79\% laser power. C-CP film imaging utilized a beam diameter of $\sim 100 \mu \mathrm{m}$, and a $200 \mu \mathrm{m}$ raster, recording spectra at every other coordinate position (e.g., 1, 3, 5, etc.). Spectral background (B) and noise (N) used in calculating S/ $\mathrm{B}$ and $\mathrm{S} / \mathrm{N}$ values in Table 1 are based on the average and standard deviation of the signals in a $100 \mathrm{Da}$ mass window centered $2000 \mathrm{Da}$ below that of the respective protein molecular ions.

\section{Results and Discussion}

In order to assess the potential utility of the C-CP films as MALDI substrates, an initial comparison between the responses using a standard steel target and a cast polypropylene film was performed. In each case, $2 \mu \mathrm{L}$ of the protein test solution $(2.5 \mu \mathrm{M}$ each of cytochrome $c$ and myoglobin in $100 \mathrm{mM}$ Tris-HCl) was allowed to dry, followed by the spray application of matrix. The results of these experiments are presented in Table 1 using normalized, arbitrary units. Clearly seen is the superior ion yield of the well-developed metal target. Ablation from the flat PP film results in a significant reduction in analyte ion intensities, though the film-ablated protein responses do show better signal-to-background (S/B) values. The lower protein ion yields from the polymer film are likely due to a number of reasons, including photon penetration/absorption into the polymer, differences in the extraction height (film is $0.5 \mathrm{~mm}$ above the metal target), and distortions of the electrostatic fields in the near surface region affecting extraction. Introduction of the channel structure of the C-CP films results in further degradation of the observed spectral quality as depicted in the entries for cytochrome $c$ and myoglobin in the left-hand portions of Table 1 and shown in Figure 2a. In this case, there is no discernible signal for the myoglobin constituent, and so MW and S values are not reported. In addition to the reduced ablation/ionization efficiencies described for the cast PP film substrate, there are likely geometric aspects limiting the incident photons reaching the channel bottoms as well as potential disturbances of the resultant plume formation. It is important to note that in each of these cases where desalting is not performed, large amounts

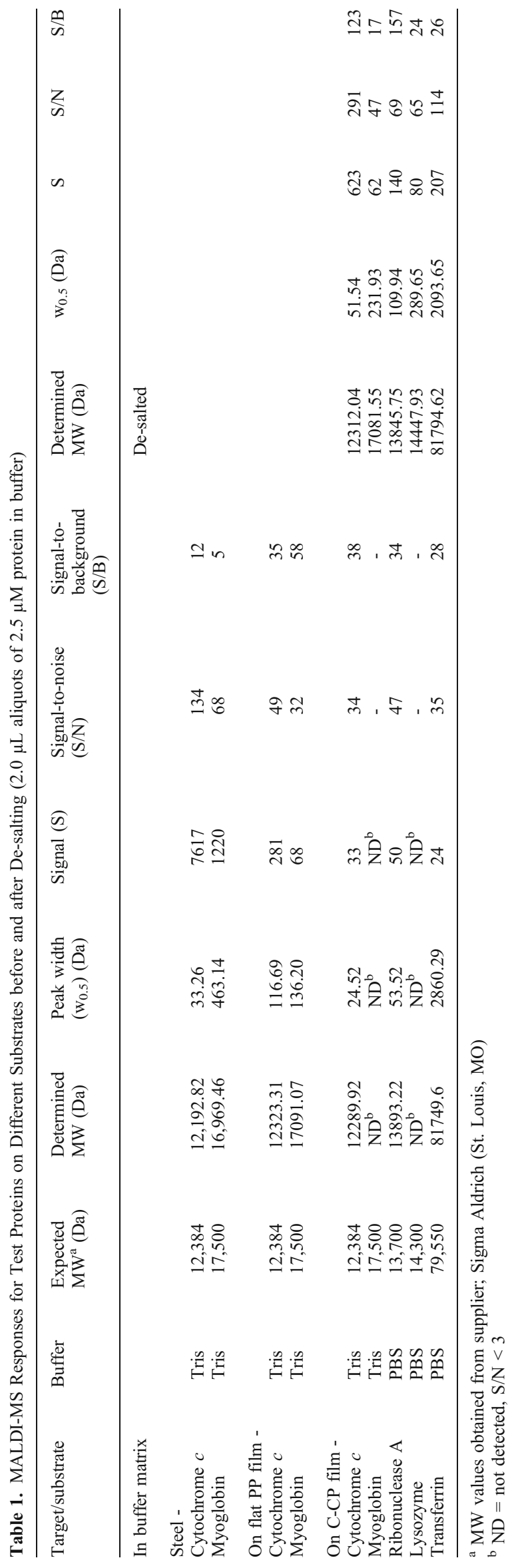



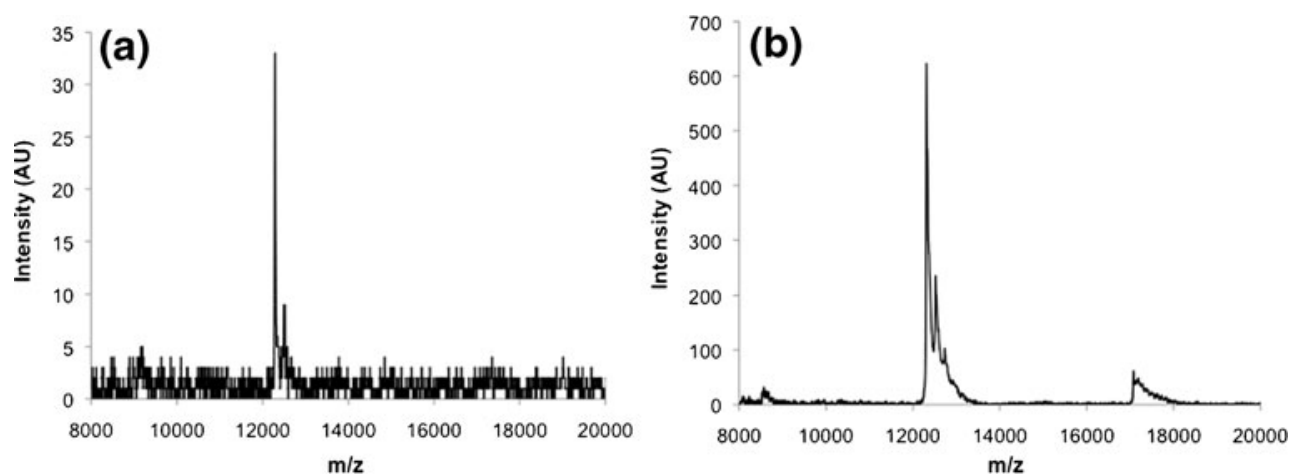

Figure 2. MALDI-MS spectra of $2 \mu \mathrm{L}$ sample aliquots containing $2.5 \mu \mathrm{M}$ (each) of cytochrome $c$ and myoglobin in $100 \mathrm{mM}$ Tris buffer deposited on a PP C-CP film; (a) as deposited and (b) following the $\mathrm{H}_{2} \mathrm{O}$ wash step

of continuum and discrete, low-mass signals are present, reflecting the presence of buffer constituents.

The ability to selectively isolate protein species within the $\mathrm{C}-\mathrm{CP}$ film channels is demonstrated in Figure 2b, with the quantitative figures of merit included in the right-hand portion of Table 1 . In this case, $9 \mu \mathrm{L}$ of $\mathrm{DI}-\mathrm{H}_{2} \mathrm{O}$ was added to the film segment after the initial solution had dried, with the solution pipetted back off the film after $5 \mathrm{~s}$ of interaction.
The proteins are selectively retained via their hydrophobic interaction with the polymer while the water-soluble buffers are removed. While the overall intensities (S) here do not match those of the metal target (Table 1), there is an appreciable improvement in $\mathrm{S}, \mathrm{S} / \mathrm{N}$, and $\mathrm{S} / \mathrm{B}$ upon completing the simple desalting step. The myoglobin signal, previously indistinguishable from spectral background values of $\sim 1 \mathrm{AU}$ shows a particularly impressive improvement of $>50 \times$. While

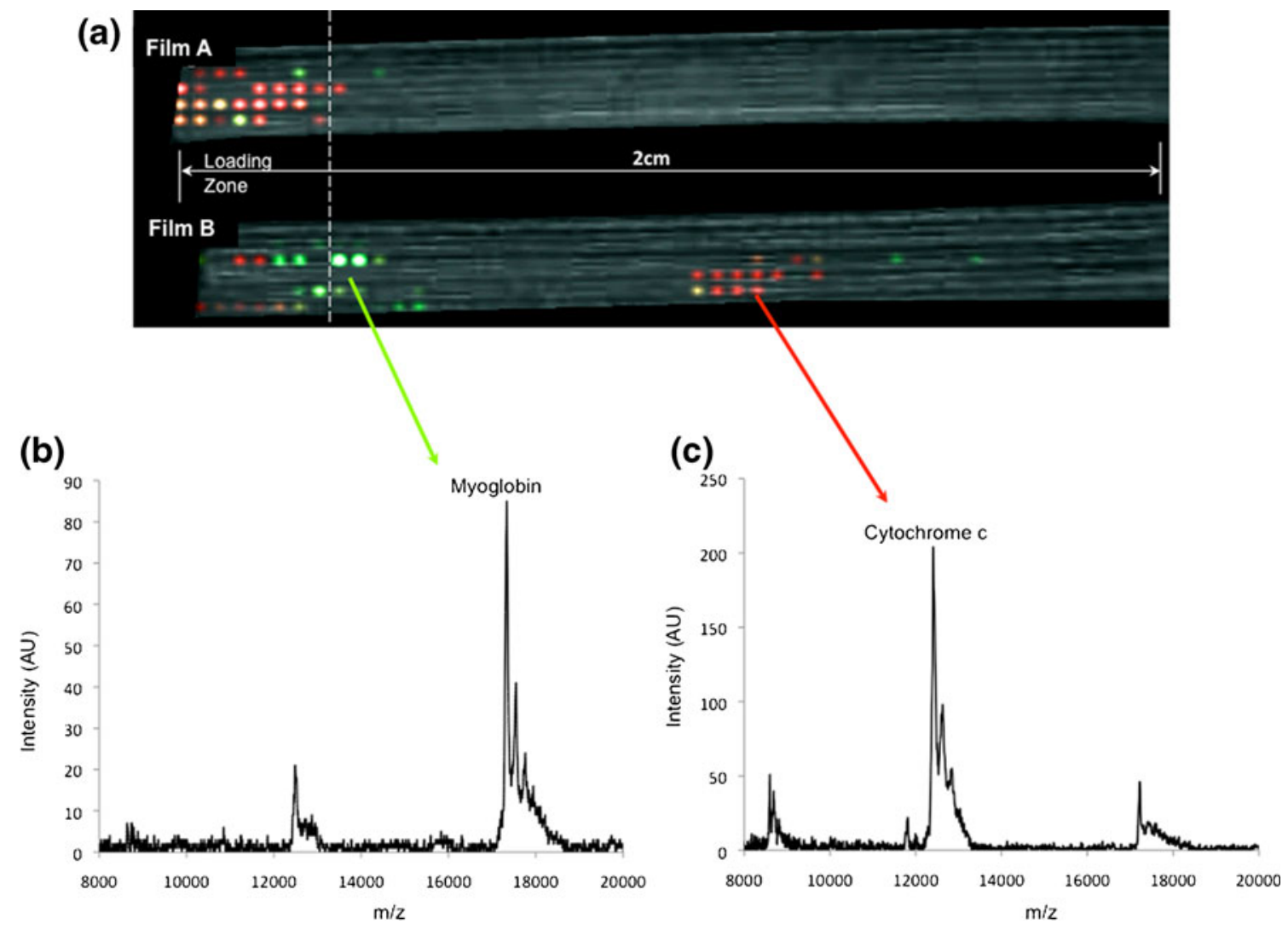

Figure 3. MALDI-MS imaging of proteins on PP C-CP film used as a chromatographic stationary phase. (a) Cytochrome $c$ and myoglobin distributions on films depicted as red and green dots, respectively, from Compass software; film $\mathrm{A}$ following the $\mathrm{H}_{2} \mathrm{O}$ wash step and film B following the chromatographic elution; (b) extracted mass spectrum representing myoglobin following elution with 50:50 ACN: $\mathrm{H}_{2} \mathrm{O}$ with $0.1 \%$ TFA; and (c) extracted mass spectrum representing cytochrome $c$ following elution with $50: 50$ ACN: $\mathrm{H}_{2} \mathrm{O}$ with $0.1 \%$ TFA 
not seen in the mass spectrum (Figure 2b), there is also a dramatic decrease in the mass-dependent continua and buffer related signals upon on-film desalting of the sample.

To further demonstrate utility of the C-CP films, three additional proteins (ribonuclease A, lysozyme, and transferrin) present in a different buffer solution, $150 \mathrm{mM}$ PBS, were evaluated before and after desalting. As presented in Table 1, the improvement in signal intensity is $\sim 3 \times$ for ribonuclease A, while that for transferrin is far more substantial. As was seen previously for myoglobin, the lysozyme signal increased from a non-detectible value of $\sim 1$ to 80 , the result of removing the suppressing salt species. Across the group of proteins, the spectral background levels do increase upon desalting, while the standard deviation of the backgrounds do not change as much. Thus, the improvements in S/N are generally better upon desalting than S/B. As would be hoped, the films' desalting abilities appear to be equally effective for these five proteins in two different buffer/salt systems.

Included in Table 1 are the determined molecular weights (MW) and respective peak widths for each of the test proteins and target materials. The peak widths for this MALDI-MS should result in resolving powers $(\mathrm{m} / \Delta \mathrm{m})$ of $\sim 1000$. In the case of the two-protein mixture in Tris buffer on the steel target, it is clear that the presence of buffer has led to an erroneous MW assignment due to adduct formation as well as pronounced peak broadening beyond what would be expected for neat protein solutions. As described above, it would not be unexpected that the insulating nature of the polymer films would perturb the extraction efficiencies of the target ions. This is seen first in the MW and peak widths for the flat PP film, and also for the C-CP films. Errors in mass accuracy could also be attributed from the fact that the mass scale was calibrated for the metal target. Mass calibration from a film target and recessing the film into the metal substrate (to have equivalent laser focus and ion extraction distances) might be expected to alleviate some of the problems. It is clear, though, that the use of the $\mathrm{C}-\mathrm{CP}$ film does not detract from the mass analyzer performance figures of merit beyond the flat film itself, though offering the capacity for greatly improved signal characteristics. One final point of relevance for transferrin is the fact that the broad peak is not unexpected for MALDI-MS of glycoproteins. In fact, the presence of intact, glycosylated protein may indicate a particular attractive feature of this method.

The second potential advantage of using $\mathrm{C}-\mathrm{CP}$ films as MALDI-MS substrates lies in the ability to achieve chromatographic separations. The realization of this aspect is demonstrated in Figure 3. Seen in Figure 4a are the MALDI-MS images of C-CP films wherein the first $3 \mathrm{~mm}$ of the film is used as an initial deposition zone. The film images appear as colored dots (red and green representing cytochrome $c$ and myoglobin, respectively), generated by the Compass software, using a $25 \%$ threshold. In film A, 2 $\mu \mathrm{L}$ of the protein:buffer solution was applied, allowed to dry, and a $9 \mu \mathrm{L}$ aliquot of DI: $\mathrm{H}_{2} \mathrm{O}$ applied to the end of the film using a syringe as described above for the short film segment. Mass spectra derived from this region are ostensibly the same as the data of Table 1, reflecting the desalting of the test solution.

As in the case of conventional TLC, spatial separation/ segregation of proteins is achieved on the $\mathrm{C}-\mathrm{CP}$ films by elution with organic solvents. In reversed-phase HPLC on PP $\mathrm{C}-\mathrm{CP}$ fiber columns, separations occur via gradient elution using an $\mathrm{ACN}$ : $\mathrm{H}_{2} \mathrm{O}$ combination containing $0.1 \%$ TFA as an ion paring agent [17]. To demonstrate the potential to affect differential mobility of the myoglobin/cytochrome $c$ pair, a simple 50:50 ACN: $\mathrm{H}_{2} \mathrm{O}$ solvent composition was chosen. As shown in the MALDI-MS image of film B in Figure 3a, as well as the extracted mass spectra of Figure $3 \mathrm{~b}$ and $\mathrm{c}$, this solvent system results in separation of the two proteins. Specifically, cytochrome $c$ is seen to elute approximately $1.1 \mathrm{~cm}$ down the film, while myoglobin, which has a greater affinity for the film surface moves only a short distance from the deposition zone. These results are in agreement with previous HPLC separations wherein cytochrome $c$ elutes at lower solvent strength than myoglobin [17].

\section{Conclusions and Future Work}

The principle of using $\mathrm{C}-\mathrm{CP}$ films as processing platforms to affect desalting and separation of proteins has been demonstrated. While there are reductions in overall sensitivity in comparison to metal target deposition, as well as some degradation of MS performance, there are many paths forward toward improving the overall response, including use of films of fewer channel number to spatially restrict the solution, detailed study of the role of channel:laser beam orientation, and modification of ion extraction parameters. By the same token, there is a wealth of opportunities in the realm of protein solution processing, both from the simple desalting perspective as well as achieving higher resolution separations. Many challenges remain, but the overall simplicity, flexibility, and practicality of the approach is believed to warrant further development and applications.

\section{Acknowledgments}

This material is based upon work supported by the National Science Foundation Division of Chemistry under grant no. 1011820 (co-funded by the MPS/CHE, ENG/CBET, and EPSCoR).

\section{References}

1. Handbook of Thin Layer Chromatography. New York: Marcel Dekker, Inc., 2003

2. Wall, P.E.: Thin-layer Chromatography: A Modern Practical Approach. Royal Society of Chemistry, Great Britian (2005)

3. Busch, K.L.: Mass-spectrometric detction for thin-layer chromatographic separations. Trac-Trends Anal. Chem. 11, 314-324 (1992)

4. Busch, K.L., Mullis, J.O., Chakel, J.A.: High resolution imaging of samples in thin layer chromatograms using a time-of-flight secondary ion mass spectrometer. J. Planar Chromatogr. Modern TLC 5, 9-15 (1992) 
5. Rogers, K., Milnes, J., Gormally, J.: The laser desorption laser ionization mass-spectra of some methylated xanthines and the laser desorption of caffeine and theophylline from thin-layer chromatography plates. Int. J. Mass Spectrom. Ion Processes 123, 125-131 (1993)

6. Scalarone, D., Duursma, M.C., Boon, J.J., Chiantore, O.: MALDI-TOF mass spectrometry on cellulosic surfaces of fresh and photo-aged Diand triterpenoid varnish resins. J. Mass Spectrom 40, 1527-1535 (2005)

7. Bakry, R., Bonn, G.K., Mair, D., Svec, F.: Monolithic porous polymer layer for the separation of peptides and proteins using thin-layer chromatography coupled with MALDI-TOF-MS. Anal. Chem. 79, 486-493 (2006)

8. Gusev, A.I., Vasseur, O.J., Proctor, A., Sharkey, A.G., Hercules, D.M.: Imaging of thin-layer chromatograms using matrix/assisted laser desorption/ionization mass-spectrometry. Anal. Chem. 67, 4565-4570 (1995)

9. Fuchs, B., Suss, R., Nimptsch, A., Schiller, J.: MALDI-TOF-MS directly combined with TLC: A review of the current state. Chromatographia 69, S95-S105 (2009)

10. Terry, D.E., Umstot, E., Desiderio, D.M.: Optimized sample-processing time and peptide recovery for the mass spectrometric analysis of protein digests. J. Am. Soc. Mass Spectrom. 15, 784-794 (2004)

11. Shukla, A., Majors, R.E.: Micropipette tip-based sample preparation for bioanalysis. $L C / G C$ 23, 646-660 (2005)

12. Duan, J.C., Wang, H., Cheng, Q.A.: On-plate desalting and SALDI-MS analysis of peptides with hydrophobic silicate nanofilms on a gold substrate. Anal. Chem. 82, 9211-9220 (2010)

13. Xu, Y.D., Bruening, M.L., Watson, J.T.: Nonspecific, on-probe cleanup methods for MALDI-MS samples. Mass Spectrom. Rev. 22, 429-440 (2003)
14. Marcus, R.K., Davis, W.C., Knippel, B.C., LaMotte, L., Hill, T.A., Perahia, D., Jenkins, D.: Capillary-channeled polymer fibers as stationary phases in liquid chromatography separations. J. Chromatogr. A 986, 17-31 (2003)

15. Nelson, D.K., Marcus, R.K.: A novel stationary phase: CapillaryChanneled Polymer (C-CP) fibers for HPLC separations of proteins. $J$. Chromatogr. Sci. 41, 475-479 (2003)

16. Stanelle, R.D., Mignanelli, M., Brown, P., Marcus, R.K.: CapillaryChanneled Polymer (C-CP) fibers as a stationary phase in microbore high-performance liquid chromatography columns. Anal. Bioanal. Chem. 384, 250-258 (2006)

17. Nelson, D.M., Marcus, R.K.: Characterization of Capillary-Channeled Polymer fiber stationary phases for high-performance liquid chromatography protein separations: Comparative analysis with a packed-bed column. Anal. Chem. 78, 8462-8471 (2006)

18. Fornea, D.S., Wu, Y., Marcus, R.K.: Capillary-Channeled Polymer fibers as a stationary phase for desalting of protein solutions for electrospray ionization mass spectrometry. Anal. Chem. 78, 5617-5621 (2006)

19. Pittman, J.J., Klep, V., Luzinov, I., Marcus, R.K.: Extraction of metals from aqueous systems employing Capillary-Channeled Polymer (C-CP) fibers modified with Poly(acrylic acid) (PAA). Anal. Methods 2, 461469 (2010)

20. Liu, J., Tseng, K., Garcia, B., Lebrilla, C.B., Mukerjee, E., Collins, S., Smith, R.: Electrophoresis separation in open microchannels. A method for coupling electrophoresis with MALDI-MS. Anal. Chem 73, 21472151 (2001) 